\title{
Pearls
}

\section{Pearls: How to Place an Interposition Segmental Bone Block for Lengthening or Alignment}

\author{
Judith F. Baumhauer MD, MPH
}

 here are many orthopaedic surgical procedures that call for the placement of bone blocks in order to gain length and/or alter alignment [1, 2, 4]. Two such operations include lateral column lengthening for correction of a flatfoot deformity, and placement of a segmental bone graft to salvage bone loss due to Charcot arthropathy or a failed joint replacement of the ankle or hallux.

Note from the Editor-in-Chief:

We are pleased to present the next installment of "Pearls", a column in Clinical Orthopaedics and Related Research ${ }^{\circledR}$. In this column, distinguished surgeons, scientists, or scholars share surgical or professional tips they use to help surmount important or interesting problems. We welcome reader feedback on all of our columns and articles; please send your comments to eic@clinorthop.org.

The author certifies that she, or any member of her immediate family, have no funding or commercial associations (eg, consultancies, stock ownership, equity interest, patent/ licensing arrangements, etc) that might pose a conflict of interest in connection with the submitted article.

All ICMJE Conflict of Interest Forms for authors and Clinical Orthopaedics and Related Research ${ }^{\mathbb{R}}$ editors and board members are on file with the publication and can be viewed on request.
Regardless of the surgery, placing these bone blocks with good interference fit and without fragmentation can be difficult. The goal is to have excellent bone-to-bone contact between the bone block graft and the host bone to allow for union. I use a simple technique that successfully places the segmental bone block without damage to the adjacent host surfaces or the bone graft, and utilizes readily available instrumentation.

Two osteotomes sized the same as the adjacent host bone surfaces are positioned against these surfaces, the bone block graft is placed between these osteotomes and manually pressed into place as far as possible using the

The opinions expressed are those of the writers, and do not reflect the opinion or policy of Clinical Orthopaedics and Related Research $^{\mathbb{R}}$ or The Association of Bone and Joint Surgeons ${ }^{\circledR}$.

J. F. Baumhauer MD, MPH ( $ه)$

University of Rochester School of

Medicine and Dentistry, 601 Elmwood

Avenue, Box 665, Rochester, NY 14642,

USA

e-mail: judy_baumhauer@

urmc.rochester.edu osteotomes as if they were functioning as a skid. The smooth surface of the osteotome allows the autograft or allograft to slide along the surface with less friction than the cancellous rough bone. At a point when the graft will not advance any further, the osteotomes ends are "pinched" together using the graft as a fulcrum to allow the osteotomes to open the deeper area and allow the graft to be advanced further and seated. A bone tamp and mallet can be used carefully, as less force is needed to advance the graft and therefore there is less chance of damage to the bone block. Once the bone block is seated, the osteotomes can be removed carefully while manually holding the bone graft to keep it in place. Fixation to stabilize the graft is performed using the individual surgeon's preference.

A good example of this technique can be seen in radiographs from a patient with peripheral neuropathy, specifically Charcot arthropathy of the ankle, and hindfoot with dissolution of the talus. The large talar defect necessitated a femoral calcar interposition graft to obtain height, stability, and alignment between the calcaneus 
and tibia. The first step is to prepare the calcaneal and tibial bone surfaces for the interposition bone block (Fig. 1A). A guide wire can be seen in the calcaneus in preparation for the intramedullary rod fixation after placement of the bone block (Fig. 1B). A guide wire is advanced to confirm the central location within the tibia and withdrawn back into the calcaneus (Fig. 1C). Next, two large osteotomes are placed on the corresponding calcaneal and tibial surfaces. A femoral calcar bone block is manually advanced between the osteotomes with digital pressure as far as possible (Fig. 2). The ends of the osteotomes are "pinched" to open the deeper surfaces of the tibia and calcaneus and the bone block is advanced further until seated. Next, the guide wire is advanced across the bone block (Fig. 3), and two additional guide wires are temporarily placed from the calcaneus through the bone block into the tibia to stabilize the bone block during reaming (Fig. 4). After reaming, the intramedullary rod is placed in the standard fashion. The bone block is stabilized with the intramedullary rod (Fig. 5A-B).

A lateral column lengthening procedure utilizes segmental bone graft placed within an osteotomy of the calcaneus. This calcaneal osteotomy is placed approximately $1-2 \mathrm{~cm}$ posterior to the calcaneocuboid joint. Osteotomes of the same width of the bone cuts are placed within the osteotomy. A K-wire is placed across the calcaneocuboid joint to stabilize the joint during bone block placement (Fig. 6). The block is placed between the osteotomes and advanced as far as possible with blunt pressure. The osteotomes are then "pinched" together to use the bone block as a fulcrum and open the deeper aspects of the osteotomy and the bone block can be advanced further (Fig. 7). Finally, the bone block in place (Fig. 8). There is no need for additional hardware due to the press fit placement of the bone block.

This procedure also can be used for Cotton opening-wedge osteotomy procedures [3] for the medial cuneiform and for bone-block segmental lengthening after great toe implant removal and conversion to a first metatarsal-phalangeal joint arthrodesis. This procedure is simple, uses commonly available osteotomes, and protects the host-bone surfaces from
Fig. 1A-C (A) Lateral ankle fluoroscopy image is shown after preparation of the tibial and calcaneal bone surfaces for segmental bone graft placement. Intramedullary guide wire is placed for eventual rod placement. (B) Harris fluoroscopy view confirming intramedullary guide wire placement in calcaneus is shown. (C) AP image of an ankle fluoroscopy image confirms central intramedullary guide wire placement in the tibial canal.
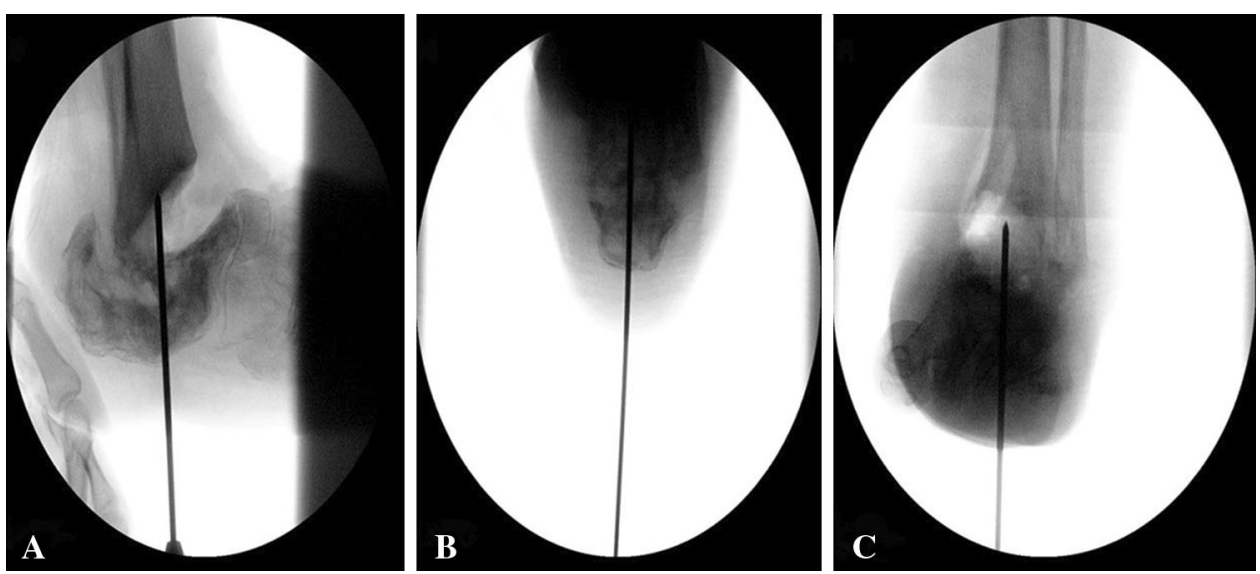


\section{Pearls}

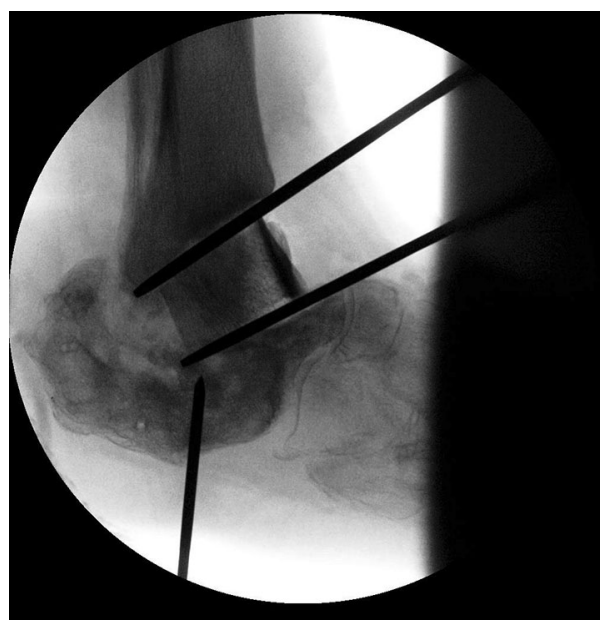

Fig. 2 Lateral ankle fluoroscopy image demonstrating placement of osteotomes-one on the tibial surface and on the calcaneal surface-allowing the segmental allograft bone to slide into place with minimal friction. The osteotomes ends can be "pinched" together allowing the deep portion of the bed to be distracted for seating of the bone block.

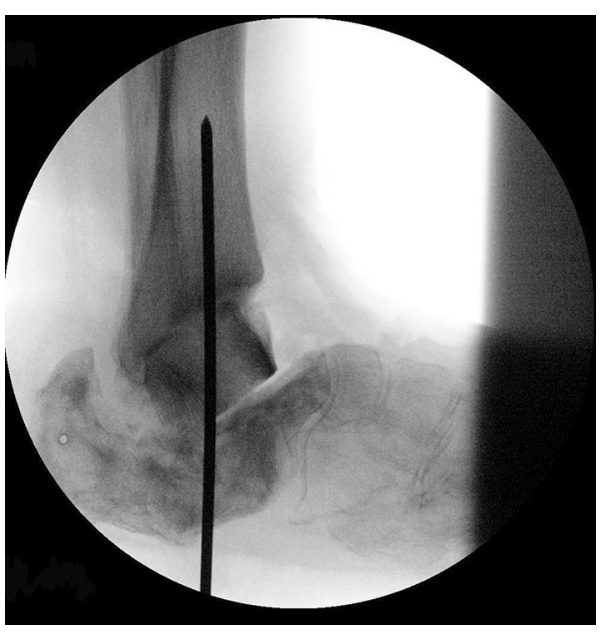

Fig. 3 Lateral ankle fluoroscopy image with the segmental bone graft in place is shown. The osteotomes are removed and the intramedullary guide wire is advanced passed the graft and into the tibia. 
Pearls

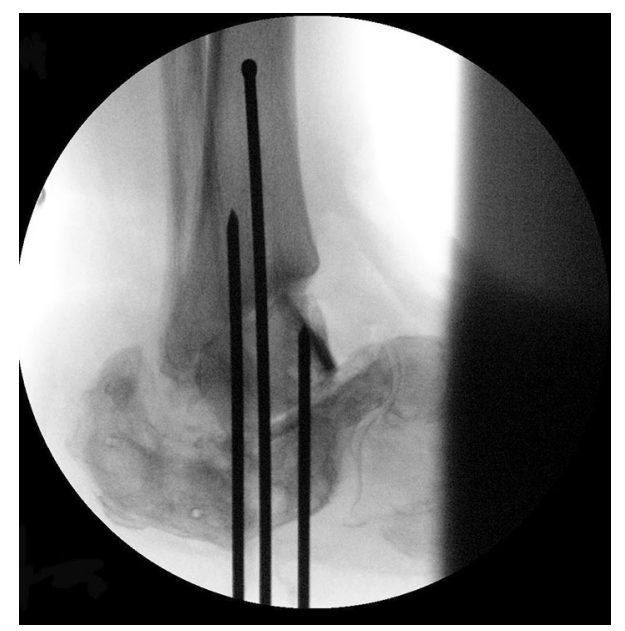

Fig. 4 Lateral ankle fluoroscopy image is shown with the ball tip guide in place in the tibia and two additional guide wires stabilizing the bone graft. This avoids displacement while reaming.
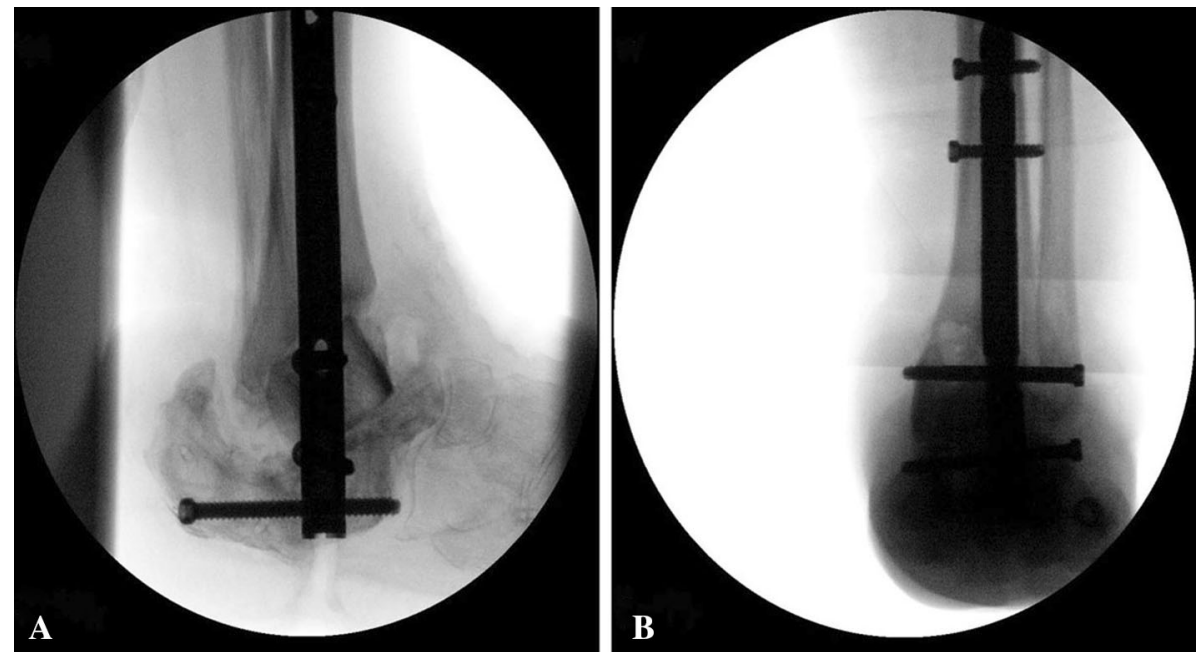

Fig. 5A-B (A) Lateral and (B) AP ankle fluoroscopy images with intramedullary nail traversing the segmental bone graft are shown. 


\section{Pearls}

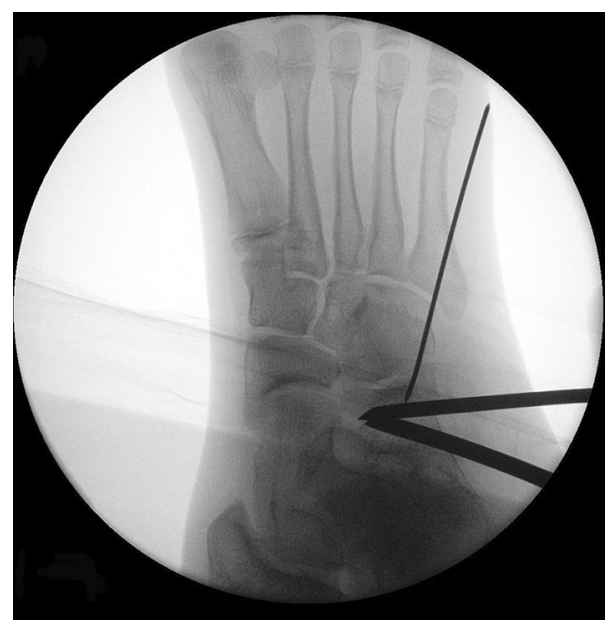

Fig. 6 Oblique foot fluoroscopy image shows a calcaneal osteotomy. One osteotome is placed on the distal cut surface and one on the proximal cut surface. There is a k-wire pinning the calcaneocuboid joint to avoid displacement of the distal calcaneal fragment.

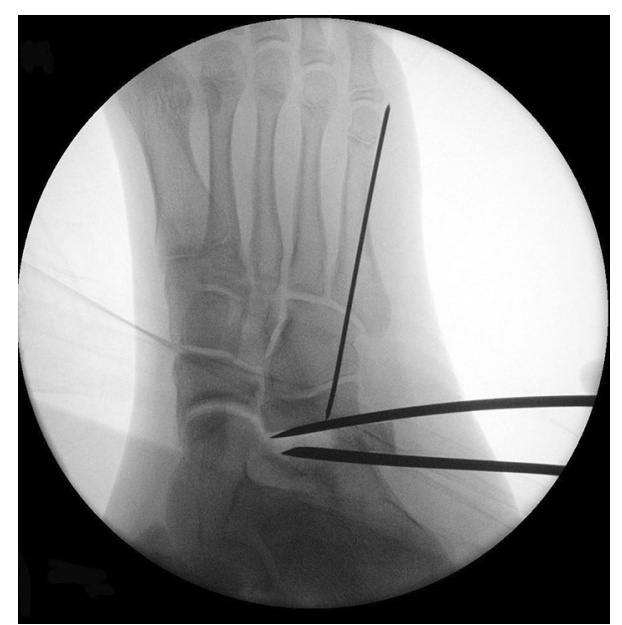

Fig. 7 Oblique foot fluoroscopy image shows the segmental bone allograft pressed into the osteotomy. At this point, the osteotomes are "pinched" together with the graft acting as a fulcrum, providing distraction of the deepest aspect of the osteotomy. The graft is advanced further into place. 


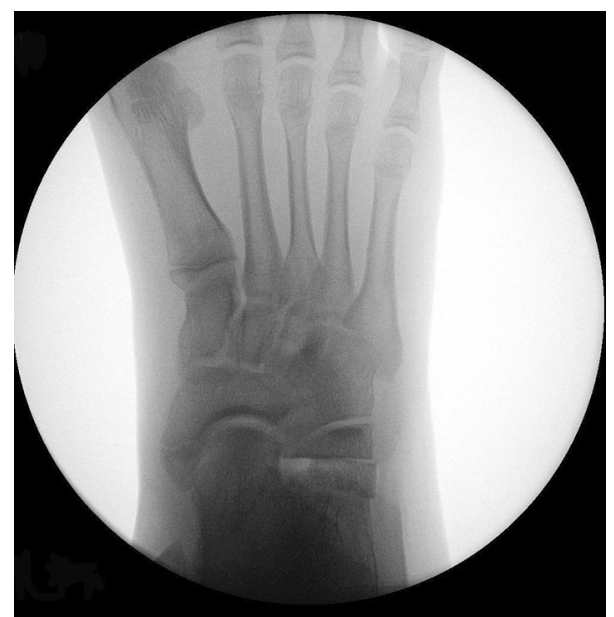

Fig. 8 Oblique foot fluoroscopy image shows the graft seated within the osteotomy. The $\mathrm{K}$-wire is removed.

compression and damage that might occur with the use of a laminar spreader. It also protects the autograft or

allograft from fragmentation, as less force is needed to advance the graft into position.

\section{References}

1. Bussewitz B, DeVries JG, Dujela M, McAlister JE, Hyer CF, Berlet GC. Retrograde intramedullary nail with femoral head allograft for large deficit tibiotalocalcaneal arthrodesis. Foot Ankle Int. 2014;35: 706-711.

2. Chan JY, Greenfield ST, Soukup DS, Do HT, Deland JT, Ellis SJ. Contribution of lateral column lengthening to correction of forefoot abduction in stage IIb adult acquired flatfoot deformity reconstruction. Foot Ankle Int. 2015;12:1400-1411.

3. Cotton FJ. Foot statistics and surgery. N Engl J Med. 1936;214:353-362.

4. Myerson MS, Schon LC, McGuigan FX, Oznur A. Result of arthrodesis of the hallux metatarsophalangeal joint using bone graft for restoration of length. Foot Ankle Int. 2000;4:297306. 